\title{
Certain Nonlinear Integral Inequalities and Their Applications
}

\author{
Yazhou Tian, ${ }^{1}$ Min Fan, ${ }^{2}$ and Yuangong Sun ${ }^{1}$ \\ ${ }^{1}$ School of Mathematical Sciences, University of Jinan, Jinan, Shandong 250022, China \\ ${ }^{2}$ Qingdao Technological University, Feixian, Shandong 273400, China
}

Correspondence should be addressed to Yazhou Tian; tianyazhou369@163.com

Received 29 September 2016; Revised 5 December 2016; Accepted 26 December 2016; Published 19 January 2017

Academic Editor: Douglas R. Anderson

Copyright (C) 2017 Yazhou Tian et al. This is an open access article distributed under the Creative Commons Attribution License, which permits unrestricted use, distribution, and reproduction in any medium, provided the original work is properly cited.

\begin{abstract}
Several new retarded integral inequalities of Gronwall-Bellman-Pachpatte type are presented which generalize the inequalities to the more general nonlinear case in the literature and provide explicit bounds on unknown functions. These results include many existing ones as special cases and can be used as tools in the qualitative analysis of certain classes of integrodifferential equations.
\end{abstract}

\section{Introduction}

It is well known that integral inequalities are very useful to investigate the existence, uniqueness, boundedness, oscillation, and other qualitative properties of solutions to differential equations and integrodifferential equations [119]. One of the fundamental inequalities is the Gronwall inequality, which was established in 1919 by Gronwall [1]. As a generalization of Gronwall inequality, Gronwall-Bellman inequality plays a key role in studying stability and asymptotic behavior of solutions to differential equations and integrodifferential equations. An important nonlinear generalization of Gronwall-Bellman inequality is Bihari's inequality [2]. In recent years, there has been much research activity concerning linear and nonlinear generalizations of GronwallBellman-Bihari type inequalities. To mention a few, Pachpatte [3] presented a new integral inequality and studied the boundedness properties of some linear integrodifferential equations, one of which we give below for the convenience of the reader.

Theorem 1 (see Pachpatte [3]). Let $u, f, g, h \in C\left(R_{+}, R_{+}\right)$, $R_{+}=[0, \infty)$, and $u_{0}$ be a nonnegative constant. If

$$
\begin{aligned}
u(t) \leq & u_{0}+\int_{0}^{t}(f(s) u(s)+h(s)) d s \\
& +\int_{0}^{t} f(s)\left(\int_{0}^{s} g(\tau) u(\tau) d \tau\right) d s,
\end{aligned}
$$

then

$$
\begin{aligned}
& u(t) \leq u_{0}+\int_{0}^{t}[h(s)+f(s) \\
& \cdot\left(u_{0} \exp \left(\int_{0}^{s}(f(\sigma)+g(\sigma)) d \sigma\right)\right. \\
& \left.\left.\quad+\int_{0}^{s} h(\sigma) \exp \left(\int_{\sigma}^{s}(f(\tau)+g(\tau)) d \tau\right) d \sigma\right)\right] d s,
\end{aligned}
$$

for $t \in R_{+}$.

Very recently, Abdeldaim and El-Deeb [4, 5] investigated the following integral inequalities which generalized the main results of [3].

Theorem 2 (see [4, Theorem 2.1]). Let $u, f, g, h \in C\left(R_{+}, R_{+}\right)$ and suppose that $\alpha \in C^{1}\left(R_{+}, R_{+}\right)$are increasing functions with $\alpha(t) \leq t$ and $\alpha(0)=0$, for $t \in R_{+}$, and $u_{0}$ is a nonnegative constant. If

$$
\begin{aligned}
u(t) \leq & u_{0}+\int_{0}^{\alpha(t)}(f(s) u(s)+h(s)) d s \\
& +\int_{0}^{\alpha(t)} f(s)\left(\int_{0}^{s} g(\tau) u(\tau) d \tau\right) d s,
\end{aligned}
$$

then

$$
\begin{gathered}
u(t) \leq u_{0}+\int_{0}^{t}\left(\alpha^{\prime}(s) h(\alpha(s))+\alpha^{\prime}(s) f(\alpha(s))\right. \\
\cdot \exp \left(\int_{0}^{\alpha(s)}(f(\tau)+g(\tau)) d \tau\right)\left(u_{0}\right.
\end{gathered}
$$




$$
\left.\left.+\int_{0}^{\alpha(s)} h(\sigma) \exp \left(-\int_{0}^{\sigma}(f(\tau)+g(\tau)) d \tau\right) d \sigma\right)\right) d s
$$

$\forall t \in R_{+}$.

Theorem 3 (see [4, Theorem 2.2] and [5, Theorem 2.1]). Let $u, f, g, h \in C\left(R_{+}, R_{+}\right)$and assume that $\varphi, \varphi^{\prime}, \alpha \in C^{1}\left(R_{+}, R_{+}\right)$ are increasing functions with $\varphi^{\prime} \leq k, \varphi>0, \alpha(t) \leq t$, and $\alpha(0)=0$, for $t \in R_{+}$, and $u_{0}$ and $k$ are positive constants. If

$u(t)$

$$
\begin{aligned}
\leq & u_{0}+\int_{0}^{\alpha(t)} \varphi(u(s))(f(s) \varphi(u(s))+h(s)) d s \\
& +\int_{0}^{\alpha(t)} \varphi(u(s)) f(s)\left(\int_{0}^{s} g(\tau) \varphi(u(\tau)) d \tau\right) d s,
\end{aligned}
$$

then

$$
u(t) \leq \Phi^{-1}\left(\Phi\left(u_{0}\right)+\int_{0}^{\alpha(t)}(h(s)+f(s) \beta(s)) d s\right)
$$

$\forall t \in R_{+}$,

where

$$
\begin{aligned}
& \beta(t) \\
& \leq \frac{\exp \left(\int_{0}^{\alpha(t)}(k h(s)+g(s)) d s\right)}{\left(\varphi^{-1}\left(u_{0}\right)-\int_{0}^{\alpha(t)} k f(s) \exp \left(\int_{0}^{s}(k h(\sigma)+g(\sigma)) d \sigma\right) d s\right)}, \\
& \Phi(r)=\int_{1}^{r} \frac{d t}{\varphi(t)}, \quad r>1,
\end{aligned}
$$

and $\Phi^{-1}$ and $\varphi^{-1}$ stand for the inverses of the functions $\Phi$ and $\varphi$, respectively.

Note that the function $\varphi$ of inequalities established in [4, 5] satisfies $\varphi^{\prime} \leq k$; that is, $\varphi$ is global Lipchitz. However, in the real system there may exist the non-Lipschitz $\varphi$, such as $\varphi(t)=t^{2}$ or $\varphi(t)=e^{t}$, and then the obtained results can not be applied to this kind of systems. The natural question now is as follows: is it possible to relax this condition? The aim of this paper is to give an affirmative answer to this question.

In this paper, we study certain classes of integral inequalities which generalize the inequalities established in [3-5] to the more general nonlinear case. The obtained results can be used as tools in the study of qualitative theory of certain classes of integrodifferential equations with more general nonlinearities. At the end, two examples are provided to illustrate the main results.

\section{Main Results}

In what follows, $R$ denotes the set of real numbers, $C(M, S)$ and $C^{1}(M, S)$ denote the sets of all continuous functions and all continuously differentiable functions defined on set $M$ with range in the set $S$, respectively, and $f^{-1}$ stands for the inverse to $f$.

The following lemmas are very useful in the proof of our main results.

Lemma 4 (see [6, Lemma 2.1]). Assume that $a \geq 0$ and $m \geq$ $n>0$. Then

$$
a^{n / m} \leq \frac{n}{m} K^{(n-m) / m} a+\frac{m-n}{m} K^{n / m}
$$

for any $K>0$.

Lemma 5 (see [7, Corollary 1]). Assume that $u, k_{i} \in$ $C\left(R_{+}, R_{+}\right), i=1,2,3$, and $a_{0}$ is a positive constant. If

$$
\begin{aligned}
u(t) \leq & a_{0}+\int_{0}^{t} k_{1}(s) u(s) d s+\int_{0}^{t} k_{2}(s) u^{2}(s) d s \\
& +\int_{0}^{t} k_{3}(s) u^{3}(s) d s,
\end{aligned}
$$

then

$$
u(t) \leq \frac{a_{0} \exp \left(\int_{0}^{t} k_{1}(s) d s\right)}{\sqrt{\left(1-a_{0} \exp \left(\int_{0}^{t} k_{1}(s) d s\right) \int_{0}^{t} k_{2}(s) d s\right)^{2}-2 a_{0}^{2} \exp \left(\int_{0}^{t} 2 k_{1}(s) d s\right) \int_{0}^{t} k_{3}(s) d s}}, \quad t \in[0, T]
$$

where $T$ is the largest number such that

$$
\begin{array}{r}
\exp \left(\int_{0}^{T} k_{1}(s) d s\right) \int_{0}^{T} k_{2}(s) d s<\frac{1}{a_{0}} \\
\frac{\exp \left(\int_{0}^{T} 2 k_{1}(s) d s\right) \int_{0}^{T} k_{3}(s) d s}{\left(1-a_{0} \exp \left(\int_{0}^{T} k_{1}(s) d s\right) \int_{0}^{T} k_{2}(s) d s\right)^{2}}<\frac{1}{2 a_{0}^{2}} .
\end{array}
$$

Theorem 6. Let $u_{0}, p, q$, and $r$ be nonnegative constants satisfying $p \geq q>0, p \geq r>0, u, f, g, h \in C\left(R_{+}, R_{+}\right)$, $\alpha \in C^{1}\left(R_{+}, R_{+}\right), \alpha^{\prime} \geq 0, \alpha(t) \leq t$, and $\alpha(0)=0$. If

$$
\begin{aligned}
u^{p}(t) \leq & u_{0}+\int_{0}^{\alpha(t)}\left(f(s) u^{q}(s)+h(s)\right) d s \\
& +\int_{0}^{\alpha(t)} f(s)\left(\int_{0}^{s} g(\tau) u^{r}(\tau) d \tau\right) d s
\end{aligned}
$$


then, for any $K>0$,

$$
\begin{gathered}
u(t) \leq\left[u_{0}+\int_{0}^{t}\left(H(s)+\frac{q}{p} K^{(q-p) / p} \alpha^{\prime}(s) f(\alpha(s))\right.\right. \\
\cdot \exp \left(\int_{0}^{\alpha(s)} J(\tau) d \tau\right) \\
\left.\left.\cdot\left(u_{0}+\int_{0}^{s} H(\sigma) \exp \left(-\int_{0}^{\alpha(\sigma)} J(\tau) d \tau\right) d \sigma\right)\right) d s\right]^{1 / p}, \\
t \in R_{+},
\end{gathered}
$$

where

$$
\begin{aligned}
& H(t)=\alpha^{\prime}(t) h(\alpha(t))+\alpha^{\prime}(t) f(\alpha(t)) \\
& \cdot\left(\frac{p-q}{p} K^{q / p}+\frac{p-r}{p} K^{r / p} \int_{0}^{\alpha(t)} g(\tau) d \tau\right), \\
& J(t)=\frac{q}{p} K^{(q-p) / p} f(t)+\frac{r}{q} K^{(r-q) / p} g(t) .
\end{aligned}
$$

Proof. Define a function $y$ by

$$
\begin{aligned}
y(t)= & u_{0}+\int_{0}^{\alpha(t)}\left(f(s) u^{q}(s)+h(s)\right) d s \\
& +\int_{0}^{\alpha(t)} f(s)\left(\int_{0}^{s} g(\tau) u^{r}(\tau) d \tau\right) d s .
\end{aligned}
$$

Then, $y(0)=u_{0}, y$ is nondecreasing, and

$$
\begin{aligned}
u(t) & \leq y^{1 / p}(t), \\
u(\alpha(t)) & \leq y^{1 / p}(\alpha(t)) \leq y^{1 / p}(t),
\end{aligned}
$$$$
t \in R_{+} \text {. }
$$

Differentiating $y$ and using (16), we conclude that

$$
\begin{aligned}
& y^{\prime}(t)=\alpha^{\prime}(t)\left[f(\alpha(t)) u^{q}(\alpha(t))+h(\alpha(t))\right]+\alpha^{\prime}(t) \\
& \cdot f(\alpha(t)) \int_{0}^{\alpha(t)} g(\tau) u^{r}(\tau) d \tau \leq \alpha^{\prime}(t) h(\alpha(t)) \\
& +\alpha^{\prime}(t) f(\alpha(t)) \\
& \cdot\left(y^{q / p}(t)+\int_{0}^{\alpha(t)} g(\tau) y^{r / p}(\tau) d \tau\right) .
\end{aligned}
$$

By virtue of Lemma 4 , for any $K>0$,

$$
\begin{aligned}
y^{q / p}(t) & \leq \frac{q}{p} K^{(q-p) / p} y(t)+\frac{p-q}{p} K^{q / p} \\
y^{r / p}(t) & \leq \frac{r}{p} K^{(r-p) / p} y(t)+\frac{p-r}{p} K^{r / p} .
\end{aligned}
$$

Combining (17) and (18), we deduce that

$$
\begin{gathered}
y^{\prime}(t) \leq H(t)+\alpha^{\prime}(t) f(\alpha(t))\left(\frac{q}{p} K^{(q-p) / p} y(t)\right. \\
\left.+\frac{r}{p} K^{(r-p) / p} \int_{0}^{\alpha(t)} g(\tau) y(\tau) d \tau\right) .
\end{gathered}
$$

Define another function $v$ by

$$
\begin{aligned}
v(t)= & \frac{q}{p} K^{(q-p) / p} y(t) \\
& +\frac{r}{p} K^{(r-p) / p} \int_{0}^{\alpha(t)} g(\tau) y(\tau) d \tau .
\end{aligned}
$$

Then $v$ is nondecreasing, $v(0)=q u_{0} K^{(q-p) / p} / p$, and

$$
\begin{aligned}
& y(t) \leq \frac{p}{q} K^{(p-q) / p} v(t), \\
& y(\alpha(t)) \leq \frac{p}{q} K^{(p-q) / p} v(\alpha(t)) \leq \frac{p}{q} K^{(p-q) / p} v(t), \\
& t \in R_{+} \text {. }
\end{aligned}
$$

It follows from (19) that

$$
y^{\prime}(t) \leq H(t)+\alpha^{\prime}(t) f(\alpha(t)) v(t)
$$

Differentiating $v$ and using (22), we obtain

$$
\begin{aligned}
& v^{\prime}(t)=\frac{q}{p} K^{(q-p) / p} y^{\prime}(t)+\frac{r}{p} K^{(r-p) / p} \alpha^{\prime}(t) g(\alpha(t)) \\
& \cdot y(\alpha(t)) \leq \frac{q}{p} K^{(q-p) / p}[H(t) \\
& \left.+\alpha^{\prime}(t) f(\alpha(t)) v(t)\right]+\frac{r}{q} K^{(r-q) / p} \alpha^{\prime}(t) g(\alpha(t)) \\
& \cdot v(t)=\frac{q}{p} K^{(q-p) / p} H(t) \\
& +\left(\frac{q}{p} K^{(q-p) / p} \alpha^{\prime}(t) f(\alpha(t))\right. \\
& \left.+\frac{r}{q} K^{(r-q) / p} \alpha^{\prime}(t) g(\alpha(t))\right) v(t) .
\end{aligned}
$$

Integrating the latter inequality from 0 to $t$, we conclude that

$$
\begin{aligned}
v(t) & \leq \frac{q}{p} K^{(q-p) / p} \exp \left(\int_{0}^{\alpha(t)} J(\tau) d \tau\right) \\
& \left(u_{0}+\int_{0}^{t} H(\sigma) \exp \left(-\int_{0}^{\alpha(\sigma)} J(\tau) d \tau\right) d \sigma\right) .
\end{aligned}
$$

Therefore,

$$
\begin{aligned}
& y^{\prime}(t) \leq H(t)+\frac{q}{p} K^{(q-p) / p} \alpha^{\prime}(t) f(\alpha(t)) \\
& \cdot \exp \left(\int_{0}^{\alpha(t)} J(\tau) d \tau\right) \\
& \cdot\left(u_{0}+\int_{0}^{t} H(\sigma) \exp \left(-\int_{0}^{\alpha(\sigma)} J(\tau) d \tau\right) d \sigma\right),
\end{aligned}
$$


which yields

$$
\begin{gathered}
y(t) \leq u_{0}+\int_{0}^{t}\left[H(s)+\frac{q}{p} K^{(q-p) / p} \alpha^{\prime}(s) f(\alpha(s))\right. \\
\cdot \exp \left(\int_{0}^{\alpha(s)} J(\tau) d \tau\right)\left(u_{0}\right. \\
\left.\left.+\int_{0}^{s} H(\sigma) \exp \left(-\int_{0}^{\alpha(\sigma)} J(\tau) d \tau\right) d \sigma\right)\right] d s .
\end{gathered}
$$

By virtue of $u(t) \leq y^{1 / p}(t)$, (13) holds. This completes the proof.

Remark 7. If $p=q=r=1$, then Theorem 6 reduces to Theorem 2.

Remark 8. If $p=q=r=1$ and $\alpha(t)=t$, then Theorem 6 reduces to Theorem 1 .

Theorem 9. Let $u, f, g, h \in C\left(R_{+}, R_{+}\right)$. Assume further that $\psi, \psi^{\prime} \in C^{1}\left(R_{+}, R_{+}\right), \psi^{\prime}>0, \psi^{\prime \prime} \geq 0, \varphi \in C^{1}\left(R_{+}, R_{+}\right), \varphi>0$,
$0 \leq \varphi^{\prime} \leq a \varphi+b, a, b \geq 0, \alpha \in C^{1}\left(R_{+}, R_{+}\right), \alpha^{\prime} \geq 0, \alpha(t) \leq t$, $\alpha(0)=0$, and $u_{0}$ is a positive constant. If, for $t \in R_{+}$,

$$
\begin{aligned}
& \psi(u(t)) \\
& \quad \leq u_{0}+\int_{0}^{\alpha(t)} \varphi(u(s))(f(s) \varphi(u(s))+h(s)) d s \\
& \quad+\int_{0}^{\alpha(t)} f(s) \varphi(u(s))\left(\int_{0}^{s} g(\tau) \varphi(u(\tau)) d \tau\right) d s,
\end{aligned}
$$

then

$$
\begin{aligned}
u(t) & \leq \psi^{-1}\left[G ^ { - 1 } \left(G\left(u_{0}\right)\right.\right. \\
& \left.\left.+\int_{0}^{\alpha(t)}\left(h(s)+f(s) \beta\left(\alpha^{-1}(s)\right)\right) d s\right)\right],
\end{aligned}
$$

where

$$
\begin{aligned}
\beta(t) & =\frac{b_{0} \exp \left(\int_{0}^{t} k_{1}(s) d s\right)}{\sqrt{\left(1-b_{0} \exp \left(\int_{0}^{t} k_{1}(s) d s\right) \int_{0}^{t} k_{2}(s) d s\right)^{2}-2 b_{0}^{2} \exp \left(\int_{0}^{t} 2 k_{1}(s) d s\right) \int_{0}^{t} k_{3}(s) d s}}, t \in[0, T], \\
k_{1}(t) & =\alpha^{\prime}(t) g(\alpha(t))+\frac{b}{\psi^{\prime}\left(\psi^{-1}\left(u_{0}\right)\right)} \alpha^{\prime}(t) h(\alpha(t)), \\
k_{2}(t) & =\frac{1}{\psi^{\prime}\left(\psi^{-1}\left(u_{0}\right)\right)} \alpha^{\prime}(t)(a h(\alpha(t))+b f(\alpha(t))), \\
k_{3}(t) & =\frac{a}{\psi^{\prime}\left(\psi^{-1}\left(u_{0}\right)\right)} \alpha^{\prime}(t) f(\alpha(t)), \\
b_{0} & =\varphi\left(\psi^{-1}\left(u_{0}\right)\right),
\end{aligned}
$$

$T$ is the largest number such that

$$
\begin{aligned}
& \exp \left(\int_{0}^{T} k_{1}(s) d s\right) \int_{0}^{T} k_{2}(s) d s<\frac{1}{b_{0}} \\
& \frac{\exp \left(\int_{0}^{T} 2 k_{1}(s) d s\right) \int_{0}^{T} k_{3}(s) d s}{\left(1-b_{0} \exp \left(\int_{0}^{T} k_{1}(s) d s\right) \int_{0}^{T} k_{2}(s) d s\right)^{2}}<\frac{1}{2 b_{0}^{2}}, \\
& G(r)=\int_{r_{0}}^{r} \frac{1}{\varphi\left(\psi^{-1}(t)\right)} d t, \quad r>r_{0}>0 .
\end{aligned}
$$

Proof. Define a function $z$ by

$z(t)$

$$
\begin{aligned}
= & u_{0}+\int_{0}^{\alpha(t)} \varphi(u(s))(f(s) \varphi(u(s))+h(s)) d s \\
& +\int_{0}^{\alpha(t)} f(s) \varphi(u(s))\left(\int_{0}^{s} g(\tau) \varphi(u(\tau)) d \tau\right) d s .
\end{aligned}
$$

Then, $z(0)=u_{0}, z$ is nondecreasing, and

$$
\begin{aligned}
& u(t) \leq \psi^{-1}(z(t)), \\
& u(\alpha(t)) \leq \psi^{-1}(z(\alpha(t))) \leq \psi^{-1}(z(t)), \\
& t \in R_{+} .
\end{aligned}
$$

It is not difficult to obtain

$$
\begin{gathered}
z^{\prime}(t)=\alpha^{\prime}(t) \varphi(u(\alpha(t)))(f(\alpha(t)) \varphi(u(\alpha(t))) \\
+h(\alpha(t)))+\alpha^{\prime}(t) f(\alpha(t)) \varphi(u(\alpha(t))) \\
\cdot \int_{0}^{\alpha(t)} g(\tau) \varphi(u(\tau)) d \tau \leq \alpha^{\prime}(t) h(\alpha(t)) \\
\cdot \varphi\left(\psi^{-1}(z(t))\right)+\alpha^{\prime}(t) f(\alpha(t)) \varphi\left(\psi^{-1}(z(t))\right)
\end{gathered}
$$




$$
\begin{aligned}
& \times\left(\varphi\left(\psi^{-1}(z(t))\right)\right. \\
& \left.+\int_{0}^{\alpha(t)} g(\tau) \varphi\left(\psi^{-1}(z(\tau))\right) d \tau\right)=\alpha^{\prime}(t) h(\alpha(t)) \\
& \cdot \varphi\left(\psi^{-1}(z(t))\right)+\alpha^{\prime}(t) f(\alpha(t)) \varphi\left(\psi^{-1}(z(t))\right) \\
& \cdot w(t)
\end{aligned}
$$

where

$$
\begin{aligned}
w(t)= & \varphi\left(\psi^{-1}(z(t))\right) \\
& +\int_{0}^{\alpha(t)} g(\tau) \varphi\left(\psi^{-1}(z(\tau))\right) d \tau
\end{aligned}
$$

$w$ is nondecreasing, $w(0)=b_{0}$, and $\varphi\left(\psi^{-1}(z(t))\right) \leq w(t)$. Differentiating $w$, we have

$$
\begin{aligned}
w^{\prime}(t)= & \varphi^{\prime}\left(\psi^{-1}(z(t))\right)\left(\psi^{-1}(z(t))\right)^{\prime} z^{\prime}(t) \\
& +\alpha^{\prime}(t) g(\alpha(t)) \varphi\left(\psi^{-1}(\mathrm{z}(\alpha(t)))\right) \\
= & \varphi^{\prime}\left(\psi^{-1}(z(t))\right) \frac{1}{\psi^{\prime}\left(\psi^{-1}(z(t))\right)} z^{\prime}(t) \\
& +\alpha^{\prime}(t) g(\alpha(t)) \varphi\left(\psi^{-1}(z(\alpha(t)))\right) .
\end{aligned}
$$

Noting that $\psi$ is increasing and $\psi^{\prime}$ is nondecreasing, we get $\psi^{\prime}\left(\psi^{-1}(z(t))\right) \geq \psi^{\prime}\left(\psi^{-1}\left(u_{0}\right)\right)$, and so

$$
\begin{aligned}
& w^{\prime}(t) \leq \frac{1}{\psi^{\prime}\left(\psi^{-1}\left(u_{0}\right)\right)}\left(a \varphi\left(\psi^{-1}(z(t))\right)+b\right) \\
& \cdot\left[\alpha^{\prime}(t) h(\alpha(t)) \varphi\left(\psi^{-1}(z(t))\right)\right. \\
& \left.+\alpha^{\prime}(t) f(\alpha(t)) \varphi\left(\psi^{-1}(z(t))\right) w(t)\right]+\alpha^{\prime}(t) \\
& \cdot g(\alpha(t)) \varphi\left(\psi^{-1}(z(t))\right) \leq \frac{1}{\psi^{\prime}\left(\psi^{-1}\left(u_{0}\right)\right)}(a w(t) \\
& +b)\left[\alpha^{\prime}(t) h(\alpha(t)) w(t)+\alpha^{\prime}(t) f(\alpha(t)) w^{2}(t)\right] \\
& +\alpha^{\prime}(t) g(\alpha(t)) w(t)=k_{1}(t) w(t)+k_{2}(t) w^{2}(t) \\
& +k_{3}(t) w^{3}(t) .
\end{aligned}
$$

Integrating the latter inequality from 0 to $t$, we have

$$
\begin{aligned}
w(t) \leq & b_{0}+\int_{0}^{t} k_{1}(s) w(s) d s+\int_{0}^{t} k_{2}(s) w^{2}(s) d s \\
& +\int_{0}^{t} k_{3}(s) w^{3}(s) d s
\end{aligned}
$$

By virtue of Lemma 5,

$$
w(t) \leq \beta(t), \quad t \in[0, T] .
$$

This and (33) imply that

$$
\begin{aligned}
z^{\prime}(t) \leq & \alpha^{\prime}(t) h(\alpha(t)) \varphi\left(\psi^{-1}(z(t))\right) \\
& +\alpha^{\prime}(t) f(\alpha(t)) \varphi\left(\psi^{-1}(z(t))\right) \beta(t),
\end{aligned}
$$

and thus

$$
\begin{aligned}
\frac{z^{\prime}(t)}{\varphi\left(\psi^{-1}(z(t))\right)} \leq & \alpha^{\prime}(t) h(\alpha(t)) \\
& +\alpha^{\prime}(t) f(\alpha(t)) \beta(t) .
\end{aligned}
$$

Integrating (40) from 0 to $t$,

$$
\begin{aligned}
& z(t) \leq G^{-1}\left(G\left(u_{0}\right)\right. \\
& \left.+\int_{0}^{\alpha(t)}\left(h(s)+f(s) \beta\left(\alpha^{-1}(s)\right)\right) d s\right),
\end{aligned}
$$

which yields (28) due to (32). The proof is complete.

Remark 10. The condition $\varphi^{\prime} \leq k$ in $[4,5]$ is relaxed to $\varphi^{\prime} \leq a \varphi+b$, where $a$ and $b$ are nonnegative constants. On the basis of this new assumption, the classes of $\varphi$ are enlarged. For example, $\varphi(t)=e^{t}$ satisfies $\varphi^{\prime} \leq a \varphi+b$ when choosing $a=1$ and $b=0$.

If we choose $\psi(u)=u^{p}, \varphi(u)=u^{q}$, and $p>q \geq 1$ in Theorem 9 , then $\varphi^{\prime}(u)=q u^{q-1}$. Letting $\Phi(u)=u^{q-1}-u^{q}$, $u>0$, we have $u^{q-1} \leq u^{q}+(1-1 / q)^{q-1} / q$, and hence $\varphi^{\prime}(u)=$ $q u^{q-1} \leq q u^{q}+(1-1 / q)^{q-1}=q \varphi(u)+(1-1 / q)^{q-1}$. Therefore, $\varphi$ satisfies $\varphi^{\prime}(u) \leq a \varphi(u)+b$ with $a=q$ and $b=(1-1 / q)^{q-1}$, and then we can obtain the following result.

Corollary 11. Assume that $u, f, g, h \in C\left(R_{+}, R_{+}\right), \alpha \in$ $C^{1}\left(R_{+}, R_{+}\right), \alpha^{\prime} \geq 0, \alpha(t) \leq t, \alpha(0)=0, u_{0}$ is a positive constant, and, for $t \in R_{+}$,

$$
\begin{aligned}
u^{p}(t) \leq & u_{0}+\int_{0}^{\alpha(t)} u^{q}(s)\left(f(s) u^{q}(s)+h(s)\right) d s \\
& +\int_{0}^{\alpha(t)} f(s) u^{q}(s)\left(\int_{0}^{s} g(\tau) u^{q}(\tau) d \tau\right) d s .
\end{aligned}
$$

Then

$$
\begin{aligned}
& u(t) \leq\left(u_{0}^{(p-q) / p}\right. \\
&\left.+\frac{p-q}{p} \int_{0}^{\alpha(t)}\left(h(s)+f(s) \beta\left(\alpha^{-1}(s)\right)\right) d s\right)^{1 /(p-q)}, \\
& t \in[0, T],
\end{aligned}
$$

where $p>q \geq 1$, 


$$
\begin{aligned}
& \beta(t)=\frac{u_{0}^{q / p} \exp \left(\int_{0}^{t} k_{1}(s) d s\right)}{\sqrt{\left(1-u_{0}^{q / p} \exp \left(\int_{0}^{t} k_{1}(s) d s\right) \int_{0}^{t} k_{2}(s) d s\right)^{2}-2 u_{0}^{2 q / p} \exp \left(\int_{0}^{t} 2 k_{1}(s) d s\right) \int_{0}^{t} k_{3}(s) d s}} \\
& k_{1}(t)=\alpha^{\prime}(t) g(\alpha(t))+\frac{1}{p}\left(1-\frac{1}{q}\right)^{q-1} u_{0}^{1 / p-1} \alpha^{\prime}(t) h(\alpha(t)), \\
& k_{2}(t)=\frac{1}{p} u_{0}^{1 / p-1} \alpha^{\prime}(t)\left(q h(\alpha(t))+\left(1-\frac{1}{q}\right)^{q-1} f(\alpha(t))\right), \\
& k_{3}(t)=\frac{q}{p} u_{0}^{1 / p-1} \alpha^{\prime}(t) f(\alpha(t)),
\end{aligned}
$$

and $T$ is the largest number such that

$$
\begin{array}{r}
\exp \left(\int_{0}^{T} k_{1}(s) d s\right) \int_{0}^{T} k_{2}(s) d s<\frac{1}{u_{0}^{q / p}} \\
\frac{\exp \left(\int_{0}^{T} 2 k_{1}(s) d s\right) \int_{0}^{T} k_{3}(s) d s}{\left(1-u_{0}^{q / p} \exp \left(\int_{0}^{T} k_{1}(s) d s\right) \int_{0}^{T} k_{2}(s) d s\right)^{2}}<\frac{1}{2 u_{0}^{2 q / p}} .
\end{array}
$$

Proof. By virtue of $\varphi(u)=u^{q}$ and $\psi(u)=u^{p}$, we obtain $\varphi\left(\psi^{-1}(u)\right)=u^{q / p}$ and

$$
\begin{aligned}
G(r) & =\int_{r_{0}}^{r} \frac{1}{\varphi\left(\psi^{-1}(t)\right)} d t=\int_{r_{0}}^{r} t^{-q / p} d t \\
& =\frac{p}{p-q}\left(r^{(p-q) / p}-r_{0}^{(p-q) / p}\right), \\
G^{-1}(r) & =\left(\frac{p-q}{p} r+r_{0}^{(p-q) / p}\right)^{p /(p-q)} .
\end{aligned}
$$

An application of Theorem 9 implies that

$$
\begin{aligned}
u(t) & \leq \psi^{-1}\left[G ^ { - 1 } \left(G\left(u_{0}\right)\right.\right. \\
& \left.\left.+\int_{0}^{\alpha(t)}\left(h(s)+f(s) \beta\left(\alpha^{-1}(s)\right)\right) d s\right)\right] \\
& =\left(u_{0}^{(p-q) / p}+\frac{p-q}{p}\right. \\
& \left.\cdot \int_{0}^{\alpha(t)}\left(h(s)+f(s) \beta\left(\alpha^{-1}(s)\right)\right) d s\right)^{1 /(p-q)} .
\end{aligned}
$$

This completes the proof.

\section{Applications}

In this section, we will use the inequalities established in Theorems 6 and 9 to study the boundedness of certain integrodifferential equations.
Example 1. Consider the following integral equation:

$$
\begin{aligned}
& u^{p}(t) \\
& =a(t) \\
& \quad+\int_{0}^{t} G\left(s, u(\alpha(s)), \int_{0}^{s} N(\tau, u(\alpha(\tau))) d \tau\right) d s, \\
& t \in R_{+},
\end{aligned}
$$

where $a(t)$ is a continuous function defined on $R^{+},|a(t)| \leq A$, $A$ is a nonnegative constant, and $G \in C\left(R_{+} \times R \times R, R\right)$ and $N \in C\left(R_{+} \times R, R\right)$ satisfy

$$
\begin{gathered}
|G(t, x, y)| \leq b(t)\left(|x|^{q}+|y|\right)+c(t), \\
|N(t, z)| \leq d(t)|z|^{r}, \quad t \in R_{+}
\end{gathered}
$$

where $p, q$, and $r$ are nonnegative constants satisfying $p \geq$ $q>0, p \geq r>0$, and $b, c, d \in C\left(R_{+}, R_{+}\right)$. Then every solution $u$ of (48) satisfies

$$
\begin{aligned}
& |u(t)|^{p} \leq A+\int_{0}^{t}\left[b(s)|u(\alpha(s))|^{q}+c(s)\right] d s+\int_{0}^{t} b(s) \\
& \cdot\left(\int_{0}^{s} d(\tau)|u(\alpha(\tau))|^{r} d \tau\right) d s=A \\
& \quad+\int_{0}^{\alpha(t)} \frac{1}{\alpha^{\prime}\left(\alpha^{-1}(s)\right)}\left[b\left(\alpha^{-1}(s)\right)|u(s)|^{q}\right. \\
& \left.\quad+c\left(\alpha^{-1}(s)\right)\right] d s \\
& \quad+\int_{0}^{\alpha(t)} \frac{b\left(\alpha^{-1}(s)\right)}{\alpha^{\prime}\left(\alpha^{-1}(s)\right)}\left(\int_{0}^{s} \frac{d\left(\alpha^{-1}(\tau)\right)}{\alpha^{\prime}\left(\alpha^{-1}(\tau)\right)}|u(\tau)|^{r} d \tau\right) d s .
\end{aligned}
$$

Now, using Theorem 6 , we obtain that, for any $K>0$,

$$
\begin{gathered}
|u(t)| \leq\left[A+\int_{0}^{t}\left(H(s)+\frac{q}{p} K^{(q-p) / p} b(s)\right.\right. \\
\cdot \exp \left(\int_{0}^{s} \widetilde{J}(\tau) d \tau\right)
\end{gathered}
$$




$$
\begin{array}{r}
\left.\left.\cdot\left(A+\int_{0}^{s} H(\sigma) \exp \left(-\int_{0}^{\sigma} \tilde{J}(\tau) d \tau\right) d \sigma\right)\right) d s\right]^{1 / p}, \\
t \in R_{+}
\end{array}
$$

where

$$
\begin{aligned}
& H(t) \\
& =c(t) \\
& +b(t)\left(\frac{p-q}{p} K^{q / p}+\frac{p-r}{p} K^{r / p} \int_{0}^{t} d(\tau) d \tau\right), \\
& \widetilde{J}(t)=\frac{q}{p} K^{(q-p) / p} b(t)+\frac{r}{q} K^{(r-q) / p} d(t),
\end{aligned}
$$

which illustrates that the solution of (48) is global boundedness.

Example 2. Consider the integrodifferential equation

$$
\begin{array}{r}
\left(u^{p}(t)\right)^{\prime}=F\left(t, u(\alpha(t)), \int_{0}^{t} H(\tau, u(\alpha(\tau))) d \tau\right), \\
u^{p}(0)=C, \quad t \in R_{+},
\end{array}
$$

where $C \neq 0$ is a constant, $F \in C\left(R_{+} \times R \times R, R\right)$, and $H \in$ $C\left(R_{+} \times R, R\right)$.

Assume that

$$
\begin{aligned}
|F(t, U, V)| & \leq|U|^{q}\left[f(t)\left(|U|^{q}+|V|\right)+h(t)\right], \\
|H(t, W)| & \leq g(t)|W|^{q}, \quad t \in R_{+},
\end{aligned}
$$

where $f, g, h \in C\left(R_{+}, R_{+}\right)$. Then the solution $u$ of (53) satisfies

$$
\begin{aligned}
& u^{p}(t) \\
& =C \\
& \quad+\int_{0}^{t} F\left(s, u(\alpha(s)), \int_{0}^{s} H(\tau, u(\alpha(\tau))) d \tau\right) d s,
\end{aligned}
$$

and hence

$$
\begin{aligned}
& |u(t)|^{p} \leq|C|+\int_{0}^{t}|u(\alpha(s))|^{q}\left[f(s)|u(\alpha(s))|^{q}\right. \\
& +h(s)] d s+\int_{0}^{t} f(s)|u(\alpha(s))|^{q} \\
& \cdot\left(\int_{0}^{s} g(\tau)|u(\alpha(\tau))|^{q} d \tau\right) d s=|C| \\
& +\int_{0}^{\alpha(t)} \frac{|u(s)|^{q}}{\alpha^{\prime}\left(\alpha^{-1}(s)\right)}\left[f\left(\alpha^{-1}(s)\right)|u(s)|^{q}\right. \\
& \left.+h\left(\alpha^{-1}(s)\right)\right] d s+\int_{0}^{\alpha(t)} \frac{f\left(\alpha^{-1}(s)\right)}{\alpha^{\prime}\left(\alpha^{-1}(s)\right)}|u(s)|^{q} \\
& +\left(\int_{0}^{s} \frac{g\left(\alpha^{-1}(\tau)\right)}{\alpha^{\prime}\left(\alpha^{-1}(\tau)\right)}|u(\tau)|^{q} d \tau\right) d s .
\end{aligned}
$$

A suitable application of Corollary 11 to (56) yields

$$
\begin{aligned}
& |u(t)| \leq\left(|C|^{(p-q) / p}+\frac{p-q}{p}\right. \\
& \cdot \int_{0}^{\alpha(t)} \frac{1}{\alpha^{\prime}\left(\alpha^{-1}(s)\right)}\left(h\left(\alpha^{-1}(s)\right)\right. \\
& \left.\left.+f\left(\alpha^{-1}(s)\right) \beta\left(\alpha^{-1}(s)\right)\right) d s\right)^{1 /(p-q)} \\
& \quad=\left(|C|^{(p-q) / p}+\frac{p-q}{p} \int_{0}^{t}(h(s)\right. \\
& \quad+f(s) \beta(s)) d s)^{1 /(p-q)}, \quad t \in[0, T],
\end{aligned}
$$

where

$$
\begin{aligned}
& \beta(t)=\frac{|C|^{q / p} \exp \left(\int_{0}^{t} k_{1}(s) d s\right)}{\sqrt{\left(1-|C|^{q / p} \exp \left(\int_{0}^{t} k_{1}(s) d s\right) \int_{0}^{t} k_{2}(s) d s\right)^{2}-2|C|^{2 q / p} \exp \left(\int_{0}^{t} 2 k_{1}(s) d s\right) \int_{0}^{t} k_{3}(s) d s}}, \\
& k_{1}(t)=g(t)+\frac{1}{p}\left(1-\frac{1}{q}\right)^{q-1}|C|^{1 / p-1} h(t), \\
& k_{2}(t)=\frac{1}{p}|C|^{1 / p-1}\left(q h(t)+\left(1-\frac{1}{q}\right)^{q-1} f(t)\right), \\
& k_{3}(t)=\frac{q}{p}|C|^{1 / p-1} f(t)
\end{aligned}
$$


and $T$ is the largest number such that

$$
\begin{aligned}
& \exp \left(\int_{0}^{T} k_{1}(s) d s\right) \int_{0}^{T} k_{2}(s) d s<\frac{1}{|C|^{q / p}}, \\
& \frac{\exp \left(\int_{0}^{T} 2 k_{1}(s) d s\right) \int_{0}^{T} k_{3}(s) d s}{\left(1-|C|^{q / p} \exp \left(\int_{0}^{T} k_{1}(s) d s\right) \int_{0}^{T} k_{2}(s) d s\right)^{2}} \\
& <\frac{1}{2|C|^{2 q / p}} .
\end{aligned}
$$

\section{Competing Interests}

The authors declare that they have no competing interests.

\section{Acknowledgments}

This work was supported by the National Natural Science Foundation of China (61174217, 61374074, 61473133, and 11671227) and the Doctoral Scientific Research Foundation of University of Jinan (1008398).

\section{References}

[1] T. H. Gronwall, "Note on the derivatives with respect to a parameter of the solutions of a system of differential equations," Annals of Mathematics. Second Series, vol. 20, no. 4, pp. 292-296, 1919.

[2] I. Bihari, "A generalization of a lemma of Bellman and its application to uniqueness problems of differential equations," Acta Mathematica Academiae Scientiarum Hungaricae, vol. 7, pp. 81-94, 1956.

[3] B. G. Pachpatte, Inequalities for Differential and Integral Equations, vol. 197 of Mathematics in Science and Engineering, Academic Press, San Diego, Calif, USA, 1998.

[4] A. Abdeldaim and A. A. El-Deeb, "On generalized of certain retarded nonlinear integral inequalities and its applications in retarded integro-differential equations," Applied Mathematics and Computation, vol. 256, pp. 375-380, 2015.

[5] A. Abdeldaim and A. A. El-Deeb, "On some generalizations of certain retarded nonlinear integral inequalities with iterated integrals and an application in retarded differential equation," Journal of the Egyptian Mathematical Society, vol. 23, no. 3, pp. 470-475, 2015.

[6] F. Jiang and F. Meng, "Explicit bounds on some new nonlinear integral inequalities with delay," Journal of Computational and Applied Mathematics, vol. 205, no. 1, pp. 479-486, 2007.

[7] W.-S. Wang, X. Zhou, and Z. Guo, "Some new retarded nonlinear integral inequalities and their applications in differentialintegral equations," Applied Mathematics and Computation, vol. 218, no. 21, pp. 10726-10736, 2012.

[8] R. Bellman, "The stability of solutions of linear differential equations," Duke Mathematical Journal, vol. 10, pp. 643-647, 1943.

[9] Y. Tian, M. Fan, and F. Meng, "A generalization of retarded integral inequalities in two independent variables and their applications," Applied Mathematics and Computation, vol. 221, pp. 239-248, 2013.
[10] Y. Tian, Y. Cai, L. Li, and T. Li, "Some dynamic integral inequalities with mixed nonlinearities on time scales," Journal of Inequalities and Applications, vol. 2015, article 12, pp. 1-10, 2015.

[11] C. Zhang, T. Li, B. Sun, and E. Thandapani, "On the oscillation of higher-order half-linear delay differential equations," Applied Mathematics Letters, vol. 24, no. 9, pp. 1618-1621, 2011.

[12] W. N. Li and W. H. Sheng, "Some nonlinear dynamic inequalities on time scales," Proceedings of the Indian Academy of Sciences-Mathematical Sciences, vol. 117, pp. 545-554, 2007.

[13] B. Zheng, "Explicit bounds derived by some new inequalities and applications in fractional integral equations," Journal of Inequalities and Applications, vol. 2014, article no. 4, 2014.

[14] R. P. Agarwal, S. Deng, and W. Zhang, "Generalization of a retarded Gronwall-like inequality and its applications," Applied Mathematics and Computation, vol. 165, no. 3, pp. 599-612, 2005.

[15] O. Lipovan, "A retarded Gronwall-like inequality and its applications," Journal of Mathematical Analysis and Applications, vol. 252, no. 1, pp. 389-401, 2000.

[16] Y. Sun and T. Hassan, "Some nonlinear dynamic integral inequalities on time scales," Applied Mathematics and Computation, vol. 220, pp. 221-225, 2013.

[17] X. Liu, L. Zhang, P. Agarwal, and G. Wang, "On some new integral inequalities of Gronwall-Bellman-BIHari type with delay for discontinuous functions and their applications," Indagationes Mathematicae, vol. 27, no. 1, pp. 1-10, 2016.

[18] P. Agarwal and J. Choi, "Certain fractional integral inequalities associated with pathway fractional integral operators," Bulletin of the Korean Mathematical Society, vol. 53, no. 1, pp. 181-193, 2016.

[19] Q.-H. Ma and E.-H. Yang, "On some new nonlinear delay integral inequalities," Journal of Mathematical Analysis and Applications, vol. 252, no. 2, pp. 864-878, 2000. 


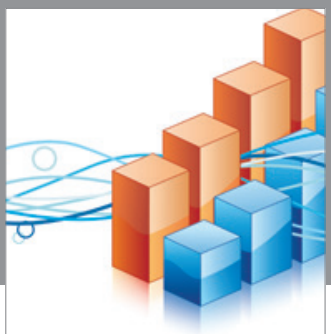

Advances in

Operations Research

vatem alat4

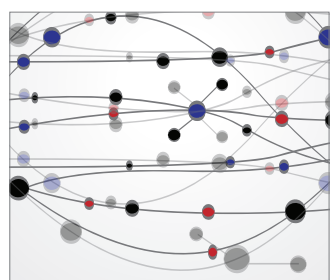

\section{The Scientific} World Journal
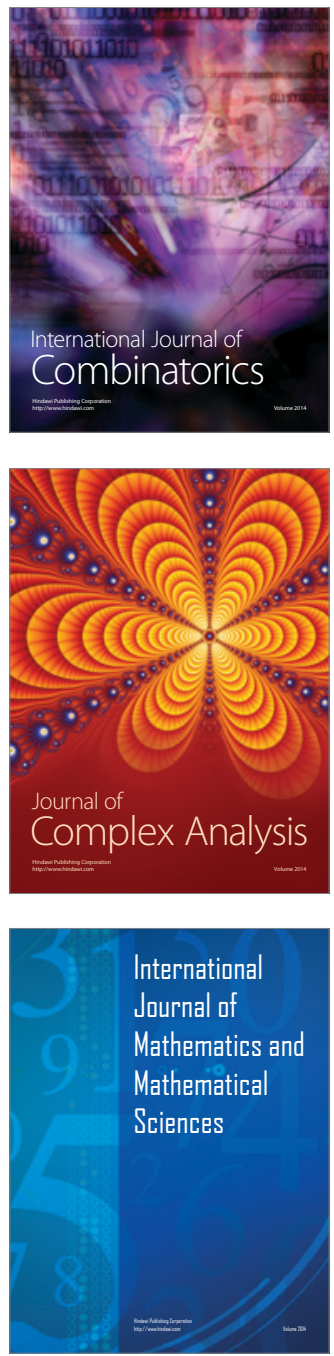
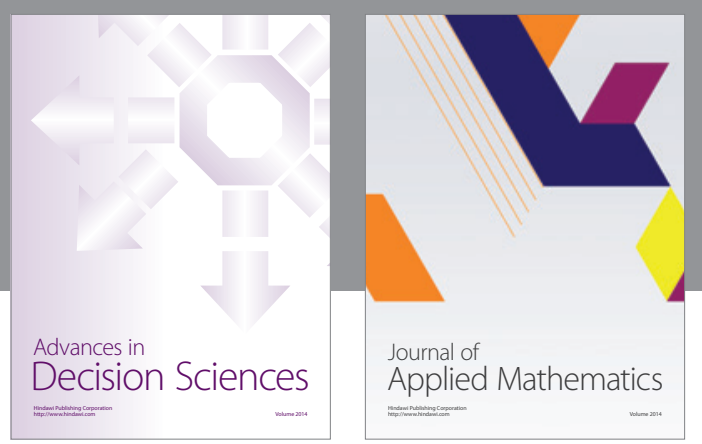

Algebra

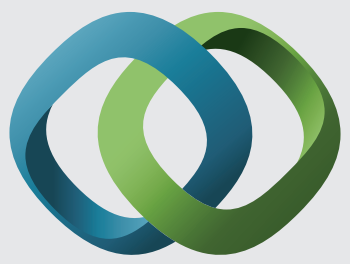

\section{Hindawi}

Submit your manuscripts at

https://www.hindawi.com
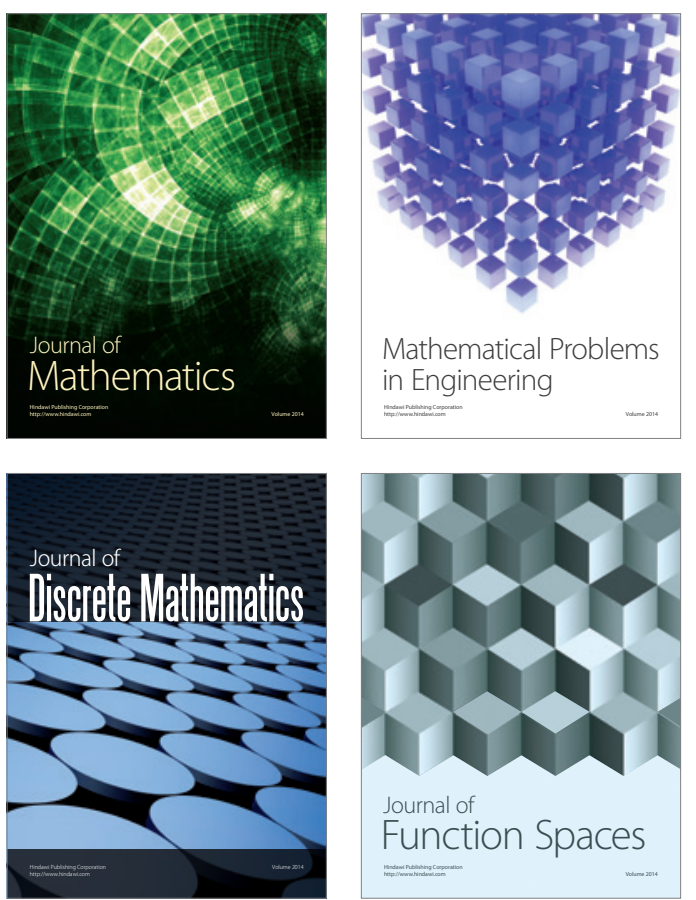

Mathematical Problems in Engineering
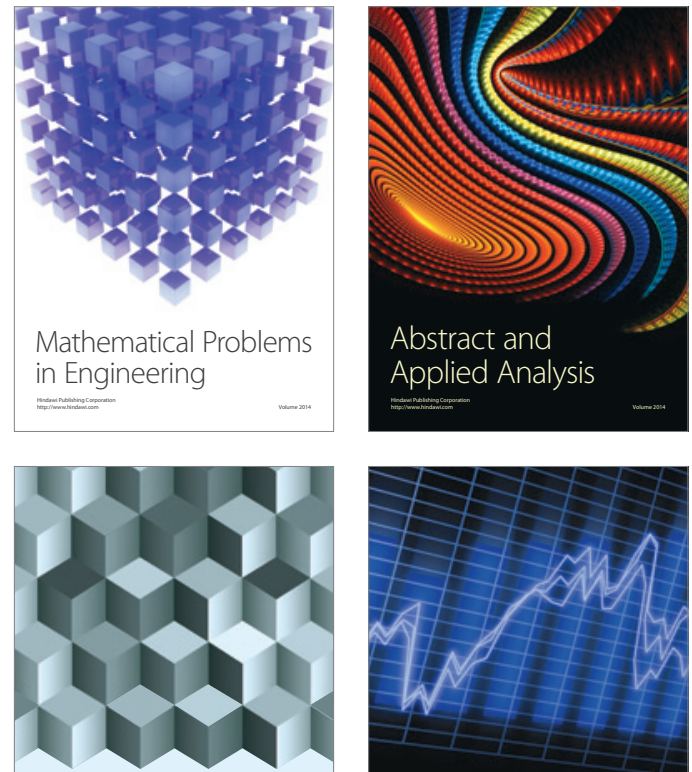

Journal of

Function Spaces

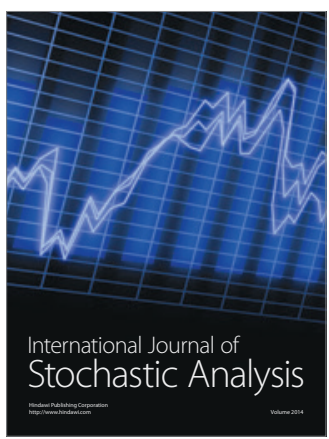

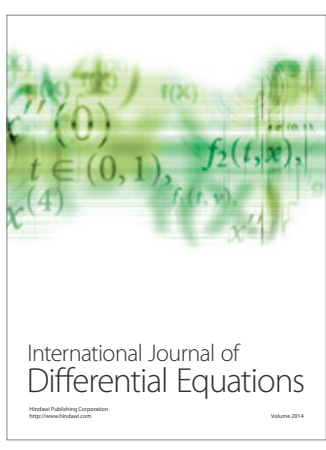
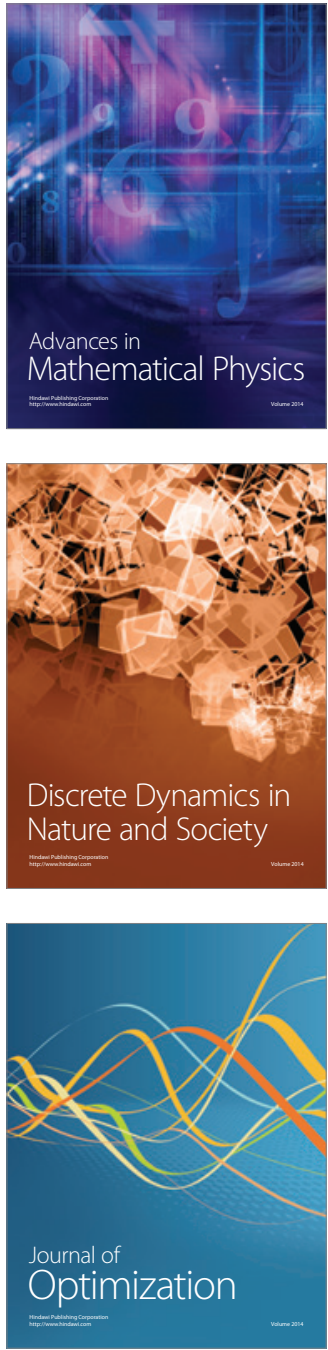